\title{
THE $F$-SIGNATURE AND STRONG $F$-REGULARITY
}

\author{
Ian M. Aberbach and Graham J. LeuschKe
}

\begin{abstract}
We show that the $F$-signature of a local ring of characteristic $p$, defined by Huneke and Leuschke, is positive if and only if the ring is strongly $F$ regular.
\end{abstract}

In [7], Huneke and Leuschke define the $F$-signature of an $F$-finite local ring of prime characteristic with perfect residue field. The $F$-signature, denoted $s(R)$, is an asymptotic measure of the proportion of $R$-free direct summands in a direct-sum decomposition of $R^{1 / p^{e}}$, the ring of $p^{e}$ th roots of $R$. This proportion seems to give subtle information on the nature of the singularity defining $R$. For example, the $F$-signature of any of the two-dimensional quotient singularities $\left(A_{n}\right),\left(D_{n}\right),\left(E_{6}\right),\left(E_{7}\right),\left(E_{8}\right)$ is the reciprocal of the order of the group $G$ defining the singularity [7, Example 18]. The main theorem of [7] on $F$-signatures is as follows.

Theorem 0.1. [7, Theorem 11] Let $(R, \mathfrak{m}, k)$ be a reduced complete F-finite Cohen-Macaulay local ring containing a field of prime characteristic $p$. Assume that $k$ is perfect. Then

(1) If $s(R)>0$, then $R$ is weakly $F$-regular.

(2) If in addition $R$ is Gorenstein, then $s(R)$ exists, and is positive if and only if $R$ is weakly F-regular.

(See below for definitions of the $F$-signature and weak $F$-regularity.)

In this note, we extend this theorem in two directions: we remove the assumption in (2) that $R$ be Gorenstein, and we replace "weakly $F$-regular" by "strongly F-regular" throughout. Our main theorem is thus as follows.

Theorem 0.2. Let $(R, \mathfrak{m}, k)$ be a reduced excellent $F$-finite local ring containing a field of characteristic $p$, and let $d=\operatorname{dim} R$. Then the following are equivalent:

(1) $\liminf \frac{a_{q}}{q^{d+\alpha(R)}}>0$.

(2) $\limsup \frac{a_{q}}{q^{d+\alpha(R)}}>0$.

(3) $R$ is strongly F-regular.

In particular, if the $F$-signature $s(R)$ is known to exist, then $s(R)$ is positive if and only if $R$ is strongly $F$-regular.

Received August 5, 2002.

The first author was partially supported by the National Science Foundation and the National Security Agency. The second author was partially supported by an NSF Postdoctoral Fellowship. 
We also extend the definition of the $F$-signature to the case of an imperfect residue field. This allows us to prove that $s(R)$ behaves well with respect to localization (Proposition 1.3).

Our results do not address the existence of the limit defining $s(R)$. Yao has shown that $s(R)$ exists whenever $R$ is Gorenstein on the punctured spectrum [10].

\section{The Main Result}

Throughout what follows, $(R, \mathfrak{m}, k)$ is a reduced Noetherian local ring of dimension $d$, containing a field of positive characteristic $p$. We use $q$ to denote a varying power of $p$. Set $d=\operatorname{dim}(R)$ and $\alpha(R)=\log _{p}\left[k: k^{p}\right]$. We assume throughout that $R$ is $F$-finite, that is, the Frobenius endomorphism $F: R \longrightarrow R$ defined by $F(r)=r^{p}$ is a module-finite ring homomorphism. Equivalently, for each $q=p^{e}, R^{1 / q}=\left\{r^{1 / q} \mid r \in R\right\}$ is a finitely generated $R$ module. In particular, this implies that $\alpha(R)<\infty$, and that $R$ is excellent [8, Propositions 1.1 and 2.5]. Also, when computing length over $R$, we have $\lambda\left(R / I^{[q]} R\right)=\lambda\left(R^{1 / q} / I R^{1 / q}\right) / q^{\alpha(R)}$.

We first define the $F$-signature of $R$.

Definition 1.1. Let $(R, \mathfrak{m}, k)$ be as above. For each $q=p^{e}$, decompose $R^{1 / q}$ as a direct sum of finitely generated $R$-modules $R^{a_{q}} \oplus M_{q}$, where $M_{q}$ has no nonzero free direct summands. The F-signature of $R$ is

$$
s(R)=\lim _{q \longrightarrow \infty} \frac{a_{q}}{q^{d+\alpha(R)}},
$$

provided the limit exists.

Our formulation differs slightly from the original definition in [7], where it is assumed that $k$ is perfect, or equivalently that $\alpha(R)=0$. This reformulation allows us to show that $s(R)$ cannot decrease upon localization. We use a lemma due to Kunz ([8]).

Lemma 1.2. Let $R$ be an $F$-finite Noetherian ring of characteristic $p$. Then for any prime ideals $P \subseteq Q$ of $R,\left[k(P): k(P)^{p}\right]=\left[k(Q): k(Q)^{p}\right] p^{\operatorname{dim} R_{Q} / P R_{Q}}$. In other words, $\alpha\left(R_{P}\right)=\alpha\left(R_{Q}\right)+$ ht $Q / P$.

Proposition 1.3. Let $(R, \mathfrak{m})$ be an $F$-finite local ring and $P$ a prime ideal. For $q=p^{e}$, let $a_{q}$ be the number of nonzero $R$-free direct summands in $R^{1 / q}$, and let $b_{q}$ be the corresponding quantity for $R_{P}$. Then

$$
\frac{b_{q}}{q^{\operatorname{dim}\left(R_{P}\right)+\alpha\left(R_{P}\right)}} \geq \frac{a_{q}}{q^{\operatorname{dim}(R)+\alpha(R)}} .
$$

In particular, if both $s(R)$ and $s\left(R_{P}\right)$ exist, then $s\left(R_{P}\right) \geq s(R)$.

Proof. We have $\left(R_{P}\right)^{1 / q} \cong\left(R^{1 / q}\right)_{P}$, so the number of $R_{P}$-free direct summands in $\left(R_{P}\right)^{1 / q}$ is at least the number of $R$-free summands in $R^{1 / q}$. A straightforward computation using Lemma 1.2 now gives the result. 
We now begin to work toward showing that $s(R)$ is positive if and only if $R$ is strongly $F$-regular. We refer the reader to [6] for basic notions concerning the theory of tight closure, including finitistic tight closure, but review briefly the ideas used in the proof.

A Noetherian ring $R$ of characteristic $p$ is said to be weakly $F$-regular provided every ideal of $R$ is tightly closed. Equivalently, the zero module is finitistically tightly closed in $E=E_{R}(k)$, the injective hull of the residue field of $R$. In other symbols, $0_{E}^{* f g}=0$. We say that $R$ is strongly $F$-regular if for every $c \in R$ not in any minimal prime of $R$, the inclusion $R c^{1 / q} \subset R^{1 / q}$ splits for $q \gg 0$. Equivalently, the zero module is tightly closed in $E$, that is, $0_{E}^{*}=0$. Weak and strong $F$-regularity are conjecturally equivalent, but this is known only in low dimension and in some special cases.

A test element for $R$ is an element $c$, not in any minimal prime of $R$, such that $c I^{*} \subseteq I$ for every ideal $I$ of $R$, and the test ideal, denoted $\tau(R)$, is the ideal generated by all test elements. For a reduced local $\operatorname{ring} R, \tau(R)=\operatorname{Ann}_{R} 0_{E}^{* f g}$ by [5, Theorem 8.23]. Thus $R$ is weakly $F$-regular if and only if $\tau(R)=R$. On the other hand, the $C S$ test ideal, cf. [9] and [2], is the ideal $\tilde{\tau}(R)=\operatorname{Ann}_{R} 0_{E}^{*}$. By work of [9] and [2], the CS test ideal behaves well under localization, so defines the non-strongly $F$-regular locus of $\operatorname{Spec}(R)$. In particular, $R$ is strongly $F$-regular if and only if $\tilde{\tau}(R)=R$.

It is known that a weakly $F$-regular ring is $F$-pure, that is, the Frobenius morphism is a pure homomorphism, and that for an $F$-pure ring both $\tau(R)$ and $\tilde{\tau}(R)$ are radical ideals.

A local ring $(R, \mathfrak{m}, k)$ is said to be approximately Gorenstein provided there is a sequence $\left\{I_{t}\right\}$ of $\mathfrak{m}$-primary irreducible ideals cofinal with the powers of $\mathfrak{m}$. When $R$ is Cohen-Macaulay and has a canonical ideal $J$ (so is Gorenstein at all associated primes), such a family can be obtained as follows: Let $x_{1}, \ldots, x_{d}$ be a system of parameters such that $x_{1} \in J$ and $x_{2}, \ldots, x_{d}$ form a system of parameters for $R / J$. Then $I_{t}:=\left(x_{1}^{t-1} J, x_{2}^{t}, \ldots, x_{d}^{t}\right) R$, for $t \geq 1$, gives the required family. Furthermore, the direct limit $\lim _{t} R / I_{t}$, where the maps in the direct system are $R / I_{t} \stackrel{x_{1} \cdots x_{d}}{\longrightarrow} R / I_{t+1}$, is isomorphic to $E_{R}(k)$. If $u_{1} \in R$ is a representative for the socle generator of $R / I_{1}$, then $u_{t}:=\left(x_{1} \cdots x_{d}\right)^{t-1} u_{1}$ generates the socle of $R / I_{t}$, and each $u_{t}$ maps in the limit to $u$, the socle element of $E_{R}(k)$.

More generally ([4, Thm. 1.7]), if $R$ is any locally excellent Noetherian ring that is locally Gorenstein at associated primes, then $R$ is approximately Gorenstein.

The following result of Hochster, together with its corollary below, explains our interest in approximately Gorenstein rings. It can be thought of as a generalization of [7, Lemma 12].

Proposition 1.4. [4, Theorem 2.6] Let $(R, \mathfrak{m})$ be an approximately Gorenstein local ring and let $\left\{I_{t}\right\}$ be a sequence of irreducible ideals cofinal with the powers 
of $\mathfrak{m}$. Let $f: R \longrightarrow M$ be a homomorphism of finitely generated $R$-modules. Then $f$ is a split injection if and only if $f \otimes_{R} R / I_{t}$ is injective for every $t$.

Proposition 1.5. Let $(R, \mathfrak{m})$ be an approximately Gorenstein local ring with a family of irreducible ideals $\left\{I_{t}\right\}$ as above, and let $u_{t} \in R$ represent a socle generator for $R / I_{t}$. Let $f: R \longrightarrow M$ be a homomorphism of finitely generated $R$-modules. If $M$ has no free summands, then there exists $t_{0}>0$ such that $u_{t} M \subseteq I_{t} M$ for all $t \geq t_{0}$.

Proof. By Proposition 1.4, $f \otimes R / I_{t}$ fails to be injective for some $t$. Since $u_{t}$ is the unique socle element of $R / I_{t}$, we have $f\left(u_{t}\right) \in I_{t} M$, that is, $u_{t} M \subseteq I_{t} M$. This continues to hold for all $t^{\prime} \geq t$, since there is an injection $R / I_{t} \longrightarrow R / I_{t^{\prime}}$ with $u_{t} \mapsto u_{t^{\prime}}$.

We also use a result of Aberbach, which says that, in some sense, elements not in tight closures are very far from being in Frobenius powers.

Theorem 1.6. [1, Prop. 2.4] Let $(R, \mathfrak{m})$ be an excellent local domain such that the completion is also a domain. Let $N=\lim _{\longrightarrow t} R / J_{t}$ be a direct limit system of cyclic modules. Fix $u \notin 0_{N}^{*}$. Then there exists $q_{0}$ such that

$$
\bigcup_{t}\left(J_{t}^{[q]}: u_{t}^{q}\right) \subseteq \mathfrak{m}^{\left[q / q_{0}\right]}
$$

for all $q \gg 0$ (where the sequence $\left\{u_{t}\right\}$ represents $u \in N$ and $u_{t} \mapsto u_{t+1}$ ).

Proof of Theorem 0.2. The Cohen-Macaulayness of $R$ is forced by the assumptions ([7, Theorem 11] and [5]), so we may assume throughout that $R$ is CohenMacaulay.

That (1) implies (2) is trivial. So assume that (2) holds. We proceed by induction on the dimension $d$, the case $d=0$ being trivial. If $d>0$, then Proposition 1.3 shows that we may assume by induction on $d$ that $R$ is strongly $F$-regular on the punctured spectrum. We will show that $0_{E}^{*}=0$, where as above $E=E_{R}(k)$ is the injective hull of the residue field of $R$.

Since $\tilde{\tau}(R)=\operatorname{Ann}_{R} 0_{E}^{*}$ is a radical ideal and is known to define the nonstrongly $F$-regular locus of $R$ (see [2]), and $R$ is strongly $F$-regular on the punctured spectrum, $\operatorname{Ann}_{R} 0_{E}^{*}$ contains the maximal ideal $\mathfrak{m}$. If $\tilde{\tau}(R)=R$, then we are done, so we assume $\tilde{\tau}(R)=\mathfrak{m}$. Then $0_{E}^{*}=\operatorname{soc}(E)$.

As in the discussion above, $E=E_{R}(k) \cong \lim _{\longrightarrow} R / I_{t}$ for a family of irreducible ideals $I_{t}$. Let $u$ be a socle generator for $E$ and $\left\{u_{t}\right\} \subseteq R$ a sequence of representatives for the socle generators of $R / I_{t}$, converging to $u$. 
Fix a power $q$ of the characteristic, and decompose $R^{1 / q} \cong R^{a_{q}} \oplus M_{q}$, where $M_{q}$ has no nonzero free summands. Then for each $t$, we have

$$
\begin{aligned}
\lambda\left(R / I_{t}^{[q]}\right)-\lambda\left(R /\left(I_{t}, u_{t}\right)^{[q]}\right)= & \frac{\lambda\left(R^{1 / q} / I_{t} R^{1 / q}\right)}{q^{\alpha(R)}-\frac{\lambda\left(R^{1 / q} /\left(I_{t}, u_{t}\right) R^{1 / q}\right)}{q^{\alpha(R)}}} \\
= & \frac{a_{q} \lambda\left(R / I_{t}\right)+\lambda\left(M_{q} / I_{t} M_{q}\right)}{q^{\alpha(R)}} \\
& \quad-\frac{a_{q} \lambda\left(R /\left(I_{t}, u_{t}\right)\right)-\lambda\left(M_{q} /\left(I_{t}, u_{t}\right) M_{q}\right)}{q^{\alpha(R)}} \\
= & \frac{a_{q} \lambda\left(R / I_{t}\right)-a_{q} \lambda\left(R /\left(I_{t}, u_{t}\right)\right)}{q^{\alpha(R)}} \\
& +\frac{\lambda\left(M_{q} / I_{t} M_{q}\right)-\lambda\left(M_{q} /\left(I_{t}, u_{t}\right) M_{q}\right)}{q^{\alpha(R)}} \\
= & \frac{a_{q}+c_{t, q}}{q^{\alpha(R)}},
\end{aligned}
$$

for some $c_{t, q} \geq 0$. By Proposition 1.5, there exists $t_{0}>0$ such that $u_{t} M_{q} \subseteq$ $I_{t} M_{q}$ for $t \geq t_{0}$, that is, $c_{t, q}=0$ for $t \geq t_{0}$. On the other hand, $\lambda\left(R / I_{t}^{[q]}\right)-$ $\lambda\left(R /\left(I_{t}, u_{t}\right)^{[q]}\right)=\lambda\left(R /\left(I_{t}^{[q]}: u_{t}^{q}\right)\right)$ is equal to 1 for large $t$ since $\left(I_{t}^{[q]}: u_{t}^{q}\right)=\mathfrak{m}$ for large $t$. Thus, for large $t$,

$$
\lim _{q \longrightarrow \infty} \frac{a_{q}+c_{t, q}}{q^{d+\alpha(R)}}=\lim _{q \longrightarrow \infty} \frac{1}{q^{d+\alpha(R)}}=0,
$$

a contradiction.

Lastly, assume that $R$ is strongly $F$-regular and keep the same notation. We then have $0_{E}^{*}=0$, so $u \notin 0_{E}^{*}$. By Theorem 1.6, then, there exists $q_{0}$ such that

$$
\left(I_{t}^{[q]}:_{R} u_{t}^{q}\right) \subseteq \mathfrak{m}^{\left[q / q_{0}\right]}
$$

for all $q \geq q_{0}$. Fix $q \geq q_{0}$. Then there exists $t_{0}$ such that for all $t \geq t_{0}$ we have

$$
\begin{aligned}
\frac{a_{q}}{q^{\alpha(R)}} & =\lambda\left(R / I_{t}^{[q]}\right)-\lambda\left(R /\left(I_{t}^{[q]}, u_{t}^{q}\right)\right) \\
& =\lambda\left(R /\left(I_{t}^{[q]}: u_{t}^{q}\right)\right) \\
& \geq \lambda\left(R / \mathfrak{m}^{\left[q / q_{0}\right]}\right) .
\end{aligned}
$$

Divide by $q^{d}$ and pass to the limit; we see that $\lim \inf \frac{a_{q}}{q^{d+\alpha(R)}} \geq \mathrm{e}_{H K}(\mathfrak{m}, R) / q_{0}^{d}>$ 0 . Thus (1) holds.

The last statement is immediate if there is a limit.

The $F$-signature suggests a form of dimension that we may attach to an $F$ finite reduced local ring. Let $s_{j}=\lim _{q \longrightarrow \infty} \frac{a_{q}}{q^{j+\alpha(R)}}$ for $0 \leq j \leq d=\operatorname{dim}(R)$ and set $s_{-1}=1$. Then we can define the $s$-dimension of $R$ as $\operatorname{sdim}(R)=$ $\max \left\{j \geq-1 \mid s_{j}>0\right\}$. A ring which is $F$-pure then has non-negative $s$-dimension, 
and Theorem 0.2 says that $R$ is strongly $F$-regular if and only if $\operatorname{sdim}(R)=$ $\operatorname{dim}(R)$.

\section{References}

[1] I. M. Aberbach, Extension of weakly and strongly F-regular rings by flat maps, J. Algebra 241 (2001), 799-807.

[2] I. M. Aberbach, F. Enescu, Test ideals and base change problems in tight closure theory, Trans. Amer. Math. Soc. 355 (2003), 619-636 (electronic).

[3] M. Hochster, Contracted ideals from integral extensions of regular rings, Nagoya Math. J. 51 (1973), 25-43.

[4] Cyclic purity versus purity in excellent Noetherian rings, Trans. Amer. Math. Soc. 231 (1977), 463-488.

[5] M. Hochster, C. Huneke, Tight closure, invariant theory, and the Briançon-Skoda theorem, J. Amer. Math. Soc. 3 (1990), 31-116.

[6] C. Huneke, Tight closure and its applications, CBMS Regional Conference Series in Mathematics, 88. American Mathematical Society, Providence, RI, 1996.

[7] C. Huneke, G. Leuschke, Two theorems about maximal Cohen-Macaulay modules, Math. Ann. 324 (2002), 391-404.

[8] E. Kunz, On Noetherian rings of characteristic p, Amer. J. Math. 98 (1976), 999-1013.

[9] G. Lyubeznik, K. E. Smith, On the commutation of the test ideal with localization and completion, Trans. Amer. Math. Soc. 353 (2001), 3149-3180 (electronic).

[10] Y. Yao, Observations on the F-signature of local rings of characteristic p, preprint.

Mathematics Department, University of Missouri, Columbia, MO 65211, U.S.A.

E-mail address: aberbach@math.missouri.edu

Department of Mathematics, University of Kansas, Lawrence, KS 66045, U.S.A.

E-mail address: gleuschke@math.ukans.edu 